\title{
A REVISED DUALITY PROOF OF SAMPLING LOCALIZATION IN RELAXATION SPECTRUM RECOVERY
}

\author{
R. J. LOY, A. R. DAVIES and R. S. ANDERSSEN ${ }^{\bowtie}$
}

(Received 21 April 2008)

\begin{abstract}
The duality proof of sampling localization given by Loy, Newbury, Anderssen and Davies in 2001 contains an oversight, as the classes of functions chosen do not assume the compact support. Here, it is shown how a minor change to the argument there yields a precise conclusion.
\end{abstract}

2000 Mathematics subject classification: 76A05, 46F12, 65R32.

Keywords and phrases: relaxation spectrum recovery, sampling localization, regularization, duality.

\section{Introduction}

To obtain information about the relaxation spectrum $H(\tau) \geq 0$ of a viscoelastic liquid one takes oscillatory shear measurements of its storage modulus

$$
G^{\prime}(\omega)=\omega \int_{0}^{\infty} G(s) \sin (\omega s) d s
$$

and its loss modulus

$$
G^{\prime \prime}(\omega)=\omega \int_{0}^{\infty} G(s) \cos (\omega s) d s
$$

where

$$
G(t)=\int_{0}^{\infty} \exp (-t / \tau) \frac{H(\tau)}{\tau} d \tau
$$

denotes the relaxation modulus. Often, it is not $H$ that is required, but rather some solution functional $[1,5]$,

$$
L_{\theta}(H)=\int_{0}^{\infty} \theta(\tau) H(\tau) d \tau,
$$

with respect to some known function $\theta$. The advantage of this approach is that, at least in theory, such functionals can be rewritten as the following equivalent data functionals

$$
L_{\theta}(H)=L_{\phi^{\prime}}\left(G^{\prime}\right)=\int_{0}^{\infty} \phi^{\prime}(\omega) G^{\prime}(\omega) d \omega
$$

(C) 2009 Australian Mathematical Society 0004-9727/2009 \$16.00 
and

$$
L_{\theta}(H)=L_{\phi^{\prime \prime}}\left(G^{\prime \prime}\right)=\int_{0}^{\infty} \phi^{\prime \prime}(\omega) G^{\prime \prime}(\omega) d \omega .
$$

In situations where, for a given function $\theta$, the corresponding $\phi^{\prime}(\omega)$ and $\phi^{\prime \prime}(\omega)$ can be determined exactly, the opportunity arises to explore theoretical properties of linear viscoelasticity. For example, when the function $\theta$ is the box-car function $B_{[a, b]}(\tau)$, which takes the value of one on the interval $[a, b]$ and is zero outside, the analytic forms of $\phi^{\prime}(\omega)$ and $\phi^{\prime \prime}(\omega)$ have been determined [1]. Consequently, these results allow one to explore the important practical situation of identifying the information required about $G^{\prime}(\omega)$ or $G^{\prime \prime}(\omega)$ in order to recover $H(\tau)$ on the interval $[a, b]$.

In [1], this is referred to as sampling localization. In [5], the authors gave an argument to support the analysis in [1]. The underlying question was to understand objects of the form

$$
\kappa_{f}=\mathcal{F}^{-1}\left(\xi_{\lambda} \cdot \widehat{f}\right),
$$

for suitable functions $f$ on $\mathbb{R}$, here $\xi_{\lambda}(r)=\cosh (\lambda r)(r \in \mathbb{R})$, for some $\lambda>0$, and $\mathcal{F}: f \mapsto \widehat{f}$ is the Fourier transform on $\mathbb{R}$. Here we choose

$$
\mathcal{F}(f)(r)=\widehat{f}(r)=\int_{-\infty}^{\infty} f(t) \exp (-i t r) d t,
$$

and

$$
\mathcal{F}^{-1}(\widehat{f})(t)=\frac{1}{2 \pi} \int_{-\infty}^{\infty} \widehat{f}(r) \exp (i t r) d r .
$$

An important aspect was to investigate the relation between the support of $f$ and the support of $\kappa_{f}$, however the latter is considered. Use was made of the classes of functions

$$
F[\lambda, p]=\left\{f \in L^{1}(\mathbb{R}) \mid \xi_{\lambda} \cdot \widehat{f} \in L^{p}(\mathbb{R})\right\},
$$

where $1 \leq p \leq 2$, for which $\kappa_{f} \in L^{q}(\mathbb{R}), 1 / p+1 / q=1$. In particular, members of these classes with compact support were used. Recently [7], it was noted that, in fact, functions in $F[\lambda, p]$ were analytic, hence zero if of compact support, and so the argument in [5] now needs to be viewed as indicative only. In the present note, we clarify the situation by first verifying the analyticity property, and then showing that with a slight change in viewpoint, the argument in [5] is valid, and establishes how the supports are related.

\section{Background}

Before progressing, it is essential to recall that measurement of an analytic function on a discrete set of points, which is all that can be achieved experimentally, does not uniquely define the analytic function even if the measurements are all exact. Values on an infinite set with a limit point are required for such determination. Consequently, in order to understand the context in which the unknown analytic function is being examined, it is necessary to invoke some approximation. 
There are numerous ways in which one can proceed, each with its advantages and disadvantages.

One possibility is to assume that some parametrized form for the unknown analytic function is known and to use the data to estimate the parameters. The advantage here is the simplicity that results by assuming the parametrized form. The disadvantage is that, in general, it is only an approximation to the unknown function.

Another possibility, which is the strategy followed in $[1,2]$, is to assume that the data is given as a function on a finite interval, and to explore the consequences of this assumption.

Renardy [7] explores the possibility that the function is known analytically, or at least can be approximated as such, and explores the use of analytic continuation. The advantage is that one can exploit the properties of analytic functions. The disadvantage, as his results confirm, is the known severe improperly posedness of analytic continuation [4, 3], in that the approximations generated behave increasingly badly as the interval on which they are assumed to be determined is extended further and further away from the limits defined in [1].

Macdonald [6] took a more traditional parametric modelling approach based on discrete Debye-type relaxations. Although this allowed approximations to $H$ to be recovered outside the limits defined in [1], they displayed a similar loss of accuracy outside these limits as Renardy's approximations.

In $[1,2,5]$, the goal was to explore the extent to which, with respect to the given finite interval, information could be recovered about the relaxation spectrum. This was achieved by approximating the box-car function $B_{[a, b]}(\tau)$ by a special class of analytic functions which approximate it arbitrarily closely. For them, the corresponding values of $\phi^{\prime}(\omega)$ and $\phi^{\prime \prime}(\omega)$ were determined analytically in [1] and used to derive related sampling localization results.

\section{Analyticity}

Suppose that $f \in L^{1}(\mathbb{R})$, and $\xi_{\lambda} \cdot \widehat{f} \in L^{p}(\mathbb{R})$. Take $s \in \mathbb{R},|s|<\lambda$. Then for any real $t$,

$$
|\exp (-i r(t+i s))|=\exp (-r s) \leq 2 \exp (|r|(s-\lambda)) \xi_{\lambda}(r),
$$

so that

$$
\int_{-\infty}^{\infty}|\widehat{f}(r) \exp (-i r(t+i s))| d r \leq 2 \int_{-\infty}^{\infty} e^{|r|(|s|-\lambda)}\left|\xi_{\lambda}(r) \widehat{f}(r)\right| d r
$$

which is finite by Hölder's inequality, since $r \mapsto e^{|r|(|s|-\lambda)}$ lies in $L^{q}(\mathbb{R})$, and

$$
\xi_{\lambda} \cdot \widehat{f} \in L^{p}(\mathbb{R})
$$

Thus,

$$
t+i s \mapsto \frac{1}{2 \pi} \int_{-\infty}^{\infty} \widehat{f}(r) e^{i r(t+i s)} d r
$$


defines an analytic function on the strip $\{t+i s:-\infty<t<\infty,|s|<\lambda\}$ about the $x$-axis, and clearly agrees with $f$ on the real axis. In particular, the only $f \in F[\lambda, p]$ of compact support is the zero function.

\section{Local behaviour of $f$ and $\kappa_{f}$}

Recall [5, (18)] that for $f \in F[\lambda, p], g \in L^{p}(\mathbb{R})$, we have to evaluate

$$
\left\langle\kappa_{f}, g\right\rangle=\lim _{\varepsilon \rightarrow 0} T_{(\varepsilon, \delta)}(f, g),
$$

where

$$
T_{(\varepsilon, \delta)}(f, g)=\sqrt{2} \pi \int_{|s|<\lambda+\delta} \frac{1}{\varepsilon} \exp \left(\frac{1}{2 \varepsilon^{2}}\left(\lambda^{2}-s^{2}\right)\right) \cos \left(\frac{\lambda s}{\varepsilon^{2}}\right)(f * g)(s) d s,
$$

with the limit in (2) being independent of the choice of $\delta>0$.

Owing to analyticity, knowledge of $f$ on any interval in fact determines $f$ everywhere, and hence $\left\langle\kappa_{f}, g\right\rangle$ for each $g \in L^{p}(\mathbb{R})$, and so $\kappa_{f} \in L^{q}(\mathbb{R})$. However, using the limit relation (2) for this evaluation obliges us to examine properties of the integral (3) for $T_{(\varepsilon, \delta)}(f, g)$.

For an interval $[a, b]$, let $c_{[a, b]}$ be an integrable function supported on $[a, b]$, and consider the effect on the right-hand side of (3) of replacing $f$ by $f+c_{[a, b]}$. Note that unless $c_{[a, b]}$ is zero the resulting function will no longer be in $F[\lambda, p]$.

Take $g \in L^{p}(\mathbb{R})$, supported outside the interval $[-b-\lambda-\delta,-a+\lambda+\delta]$ for some $\delta>0$. Then, for $|s| \leq \lambda+\delta$ and $t \in[a, b]$, we have $s-t \in[-b-\lambda-\delta$, $-a+\lambda+\delta]$ and, hence, for such $s$,

$$
\left(c_{[a, b]} \star g\right)(s)=\int_{-\infty}^{\infty} c_{[a, b]}(t) g(s-t) d t=\int_{a}^{b} c_{[a, b]}(t) g(s-t) d t=0 .
$$

Thus, the effect of $c_{[a, b]}$ on $T_{(\varepsilon, \delta)}(f, g)$ is zero, provided that $g \in L^{p}(\mathbb{R})$ is supported outside the interval $[-b-\lambda-\delta,-a+\lambda+\delta]$.

In other words, the values taken by $f$ on $[a, b]$ have no effect on $T_{(\varepsilon, \delta)}(f, g)$ for such $g$. So from (2) they have no effect on $\left\langle\kappa_{f}, f\right\rangle$ for such $g$. Since the limit in (2) is independent of $\delta>0$, there is thus no effect for $g$ supported outside $(-b-\lambda,-a+\lambda)$. That is, the effect is supported on the interval $[-b-\lambda,-a+\lambda]$.

REMARK. The interval here is the exact negative of that in [5], because of an oversight there in the order of terms in the convolution.

\section{Conclusion}

The results of [7] can be viewed as investigating how to determine $\kappa_{f}$ from values of $f$ on an arbitrary interval using other regularization methods.

The above deliberations investigate the analytic properties of a specific regularization method for determining $\kappa_{f}$ (the linear functional strategy) and 
establishes theoretically that a strict sampling localization holds if no additional information about the data than that assumed above is introduced. This does not preclude the use of alternative regularization methods that generate approximations outside the limits defined in [1]. However, the utility of such methods will depend heavily on the extent to which a chosen methodology correctly reflects additional information about the interpretation of the data outside that assumed in deriving the above strict sampling localization result.

\section{References}

[1] A. R. Davies and R. S. Anderssen, 'Sampling localization in determining the relaxation spectra', J. Non-Newt. Fluid Mech. 73 (1997), 163-179.

[2] - 'Sampling localization and duality algorithms in practice', J. Non-Newt. Fluid Mech. 79 (1998), 235-253.

[3] A. Dienstfrey and L. Greengard, 'Analytic continuation, singular-value expansions, and KramersKronig analysis', Inverse Problems 17 (2001), 1307-1320.

[4] M. M. Lavrentév, Some Improperly Posed Problems of Mathematical Physics, Springer Tracts in Nat. Philos., II (Springer, New York, 1967).

[5] R. J. Loy, C. Newbury, R. S. Anderssen and A. R. Davies, 'A duality proof of sampling localization in relaxation spectrum recovery', Bull. Aust. Math. Soc. 64 (2001), 265-269.

[6] J. R. Macdonald, 'On relaxation-spectrum estimation for decades of data: accuracy and sampling localization considerations', Inverse Problems 16 (2000), 1561-1583.

[7] M. Renardy, 'On the use of Laplace transform inversion for reconstruction of relaxation spectra', J. Non-Newt. Fluid Mech. 154 (2008), 47-51. Available online at www.sciencedirect.com/science/journal/03770257

R. J. LOY, Department of Mathematics, Mathematical Sciences Institute, Australian National University, Canberra, ACT 0200, Australia

e-mail: rick.loy@maths.anu.edu.au

A. R. DAVIES, School of Mathematics, Cardiff University, Senghennydd Road, Cardiff, CF24 4AG, Wales, UK

e-mail: daviesr@cardiff.ac.uk

R. S. ANDERSSEN, CSIRO Mathematical and Information Sciences, GPO Box 664, Canberra ACT 2601, Australia

e-mail: bob.anderssen@csiro.au 